\title{
Application of Cryo FIB SEM for Nano-EHS Studies
}

\author{
K. Scott
}

National Institute of Standards and Technology, 100 Bureau Drive, MS-8372, Gaithersburg, MD 20899

The number of consumer products incorporating engineered nanomaterials for enhanced performance and quality has increased rapidly in recent years and the concern over the health and safety implications of these nanomaterials has increased accordingly. Accurate assessment of the effects of engineered nanomaterials requires not only the careful analysis of the nanomaterials in its as-produced state but also in its biologically and environmentally relevant (in-use or productized) conditions. Physicochemical properties and behavior of nanoparticles in their productized state can be very different from their as-produced material properties [1]. For example, nanoparticles often behave like macro-particles when introduced into the natural environment due to aggregation, surface adsorbed layer formation, etc. However, the analysis of nanoparticles in their productized conditions can be challenging due to many factors such as high moisture content, opacity, poor electrical conductivity, etc. of the end product. The study of nanoparticle morphology and distribution in sunscreen is a good example of such a problem.

Studies have shown that the effectiveness (transparency and $\mathrm{UV}$ absorption) of $\mathrm{TiO}_{2}$ and $\mathrm{ZnO}$ in sunscreen depends on the particle size [2]. Also the particles have to be uniformly dispersed in sunscreen to achieve maximum UV-protection and transparency. In general, micronized titanium dioxide or zinc oxide particles used in sunscreen range from tens to several hundreds of nanometers in diameter. For accurate evaluation of particle morphology and dispersion pattern, a high resolution 3D imaging method such as focused ion beam scanning electron microscopy (FIB SEM) is needed. However, to assess the in-use nanoparticle properties, we need to analyze the nanoparticles in the emulsified condition along with the other ingredients that make up the 'lotion' and the oily ingredients used in many sunscreen formulations make it incompatible for room temperature FIB SEM analysis.

We used a cryo-FIB-SEM approach to analyze the size distribution and aggregation pattern of $\mathrm{TiO}_{2}$ and $\mathrm{ZnO}$ nanoparticles in commercially available sunscreens. A small dab of sunscreen was smeared over a copper stub and smoothed out into a thin layer using a razor blade. Next, the sample was plunge frozen in liquid nitrogen slush and transferred to the cold stage in the FIB SEM chamber under partial vacuum using a transfer device. To prevent charging and to ensure a smoothly milled surface, we 'cold' deposited a Pt layer using the method described by Hayles et al. [3] followed by rough cut trenching around the analysis volume, final polishing and serial milling and imaging of the volume of interest. Fig. 1 shows the ion beam and electron beam overview images of the prepared analysis volume. Fig. 2 represents a 3D volume rendering from one of the serial milling and imaging data sets showing large variability in particle sizes and shapes as well as many air bubbles that can complicate post processing steps. Different milling and imaging conditions were also examined for optimal sample stability and image quality as well as energy dispersive $\mathrm{x}$-ray mapping. 


\section{References}

[1] Stiller, S. et al., Colloids and Surfaces A: Physicochem. Eng. Aspects 232 (2004) 261-267.

[2] Popov, A.P. et al., J. Phys. D: Appl. Phys. 38 (2005) 2564-2570.

[3] Hayles, M.F. et al., Microsc. Microanal. 226 (2007) 263-269.

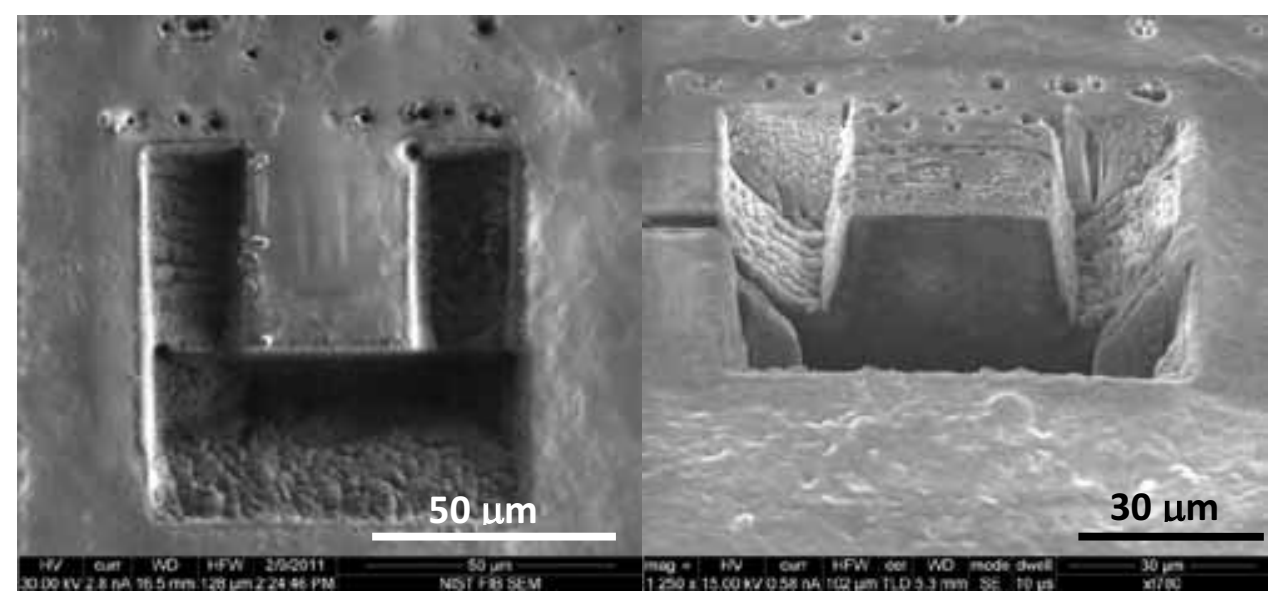

FIG. 1. Ion and electron beam images of the volume of interest. Under cryogenic conditions, redeposition rate of the milled material is high and a large amount of material around the analysis volume must be removed to prevent blockage of the analysis surface due to re-deposition.

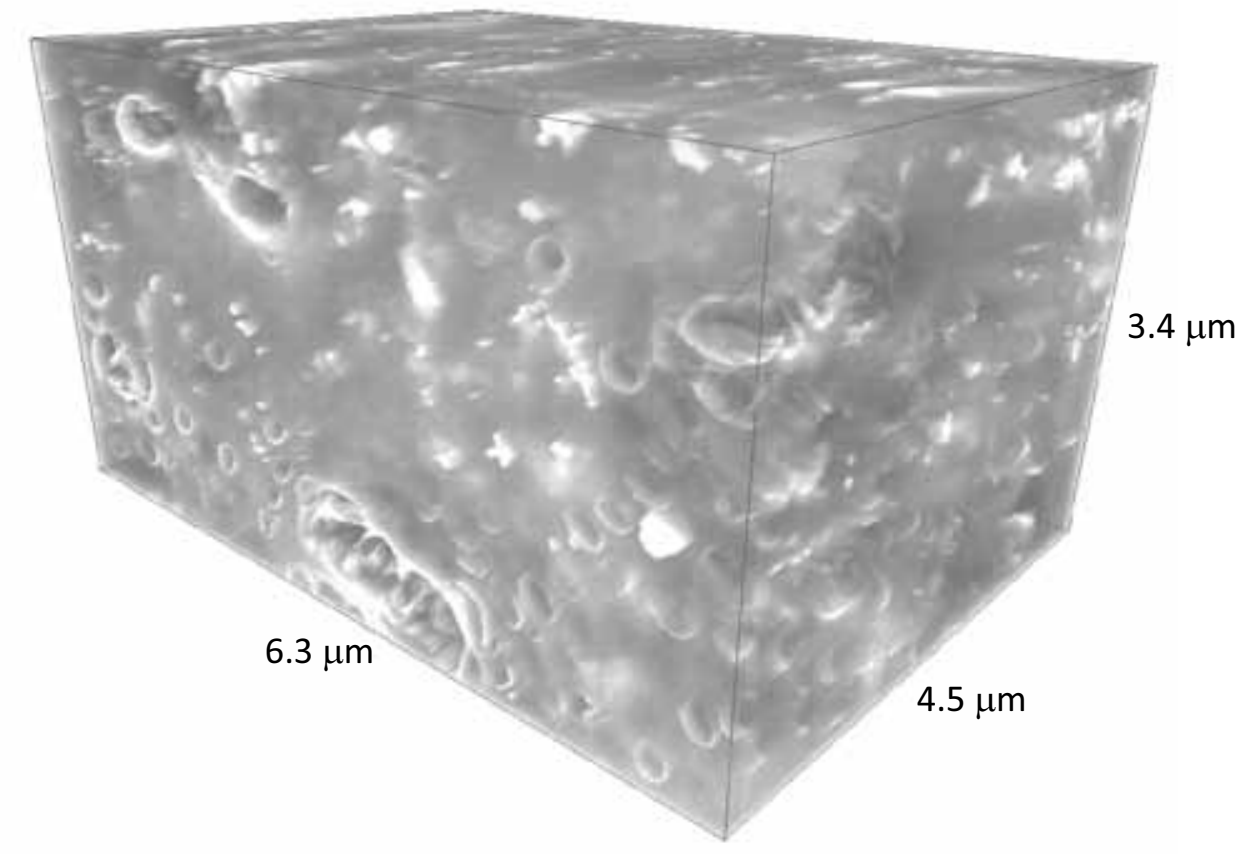

FIG. 2. 3D rendering of the analysis volume showing many air bubbles as well as varying particle sizes. This volume was generated from 90 images taken at $50 \mathrm{~nm}$ intervals. 